\title{
A Geometrical Approach for the Singularity Analysis of a 3-ㅌRS Parallel Manipulator
}

\author{
H. Tetik ${ }^{1}$ and G. Kiper ${ }^{1}$ \\ ${ }^{1}$ Department of Mechanical Engineering, Izmir Institute of Technology, Turkey, \\ e-mail: haliltetik@iyte.edu.tr;gokhankiper@iyte.edu.tr
}

\begin{abstract}
Identifying singularity manifolds of parallel manipulators analytically is a hard task due to their complex kinematics and passive joints. This study proposes to use the geometrical conditions of singularities in order to identify the singularity manifolds for a 3-ㅌRS parallel manipulator. The singularity surfaces for both inverse and forward kinematics singularities are obtained and plotted.
\end{abstract}

Key words: Parallel manipulators, Jacobian analysis, singularity analysis, singularity surfaces

\section{Introduction}

At a singular configuration of a manipulator, the end effector loses its rigidity or has uncontrollable degrees-of-freedom (dof) [5]. When considering the parallel manipulators (PM), it should be noticed that the singular configurations can be observed inside the workspace and it is highly undesirable to loose the stiffness or gain an uncontrollable dof while performing a task with the PM [9].

For the case of closed loop kinematic chains, the velocity input-output relationship is defined in [3] as $\mathbf{A} \dot{x}+\mathbf{B} \dot{\theta}=\mathbf{0}$, where $\dot{x}$ represents task space velocities and $\dot{\theta}$ represents joint space velocities. Using the velocity input-output relationship equation, 3 types of singularities are defined. The first type occurs when $\operatorname{det}(\mathbf{A})=$ 0 . For this type of singularity, the mechanism reaches either its internal or external limit of the workspace. This type of singularity is called as inverse kinematic singularity (IKS) or loss type singularity. The second type of singularity is observed when $\operatorname{det}(\mathbf{B})=0$. This type of singularty occurs within the workspace, rather than at the boundary, and it is called as forward kinematic singularities (FKS) or gain type singularities. The third type of singularity is observed when $\operatorname{det}(\mathbf{A})=\operatorname{det}(\mathbf{B})=$ 0 . This type of singularities are called as combined singularities.

The PM investigated in this study is a 3-dof manipulator which can achieve 1 dof translational and 2 dof rotational motion (1T2R). All the limbs have same kinematic structure and they are symmetrically connected to the base and moving platforms. In the literature, there are several studies investigating the singularities of PMs which have the same motion chracteristics. For a 3-PRRU PM (the P3 Robot) 
the singularity analysis is performed in [2] by evaluating the determinants of the Jacobian matrices. The singularity conditions were issued in [6] by obtaining the Jacobian matrices of a 3-PSP PM. They mention the difficulty of analytically determining the singularity equations for PMs by using the Jacobian matrices. So, instead of evaluating the Jacobian matrices, they provide the geometrical condition for the singularities. The singularities of a 3-PRS PM were investigated in [4]. Instead of evaluating the determinants of the Jacobian matrices, they investigated the geometrical conditions for the singularities of the PM. For platform type multiloop spatial mechanisms, several mechanisms were examined in [1], providing the geometrical conditions of the mechanisms at which the gain type singularities will occur.

This study investigates both IKS and FKS of a 3-RRS PM. To achieve this, firstly the geometry of the PM is explained. Then the Jacobian matrices for the inverse and forward kinematics are formulated. Using the Jacobian matrices, the singularity conditions of the PM and their physical meaning are examined. Finally, using the geometrical singularity conditions, both IKS and FKS surfaces in terms of the independent task space parameters are constructed using Mathematica ${ }^{\circledR}$ software.

\section{Manipulator Geometry}

The manipulator investigated in this study is a 3-dof PM located at Izmir Institute of Technology and it has 3 identical limbs. All the limbs have an active revolute (R) joint, a passive $\mathrm{R}$ joint and a spherical (S) joint. In Fig. 1, active R joints are shown with $O_{0 i}$, passive R joints shown with $O_{i j}$ and $\mathrm{S}$ joints are shown with $O_{7 j}$ for $i=1$, 2,3 and $j=i+3$. The $\mathrm{R}$ joint axes on each limb are parallel to each other, and therefore each limb has a planar motion. $\theta_{i}$ and $\phi_{i}$ are respectively the active and passive $\mathrm{R}$ joint variables on the $i^{\text {th }}$ link. The length of the bottom and upper links are $l_{1}=0.7 \mathrm{~m}$ and $l_{2}=0.775 \mathrm{~m}$, respectively.

Both base and platform are in the shape of an equilateral triangle with circumcircle radii $b=p=0.544 \mathrm{~m}$. The axes of the active R joints are tangential to the base circumcircle. The centers of the base and platform circumcircles are shown with $O_{0}$ and $O_{7}$, respectively. A fixed coordinate frame $\left(O_{0}-x y z\right)$ is attached to $O_{0}$ and its $x$ axis is along $\overrightarrow{O_{0} O_{01}}$ direction. A moving coordinate frame $\left(O_{7}-u v w\right)$ is attached to $O_{7}$ and its $u$ axis is along $\overrightarrow{O_{7} O_{74}}$ direction. The limbs are attached to the corners of the base and platform triangles such that $\alpha_{11}=\alpha_{44}=0^{\circ} ; \alpha_{12}\left(\angle O_{01} O_{0} O_{02}\right)=\alpha_{45}$ $\left(\angle O_{74} O_{7} O_{75}\right)=120^{\circ} ; \alpha_{13}\left(\angle O_{01} O_{0} O_{03}\right)=\alpha_{46}\left(\angle O_{74} O_{7} O_{76}\right)=240^{\circ}$.

The position of the moving platform origin $O_{7}$, with respect to $\left(O_{0}-x y z\right)$ is defined with a position vector:

$$
\vec{O}_{7}=\left[\begin{array}{lll}
O_{7, x} & O_{7, y} & O_{7, z}
\end{array}\right]^{T}
$$




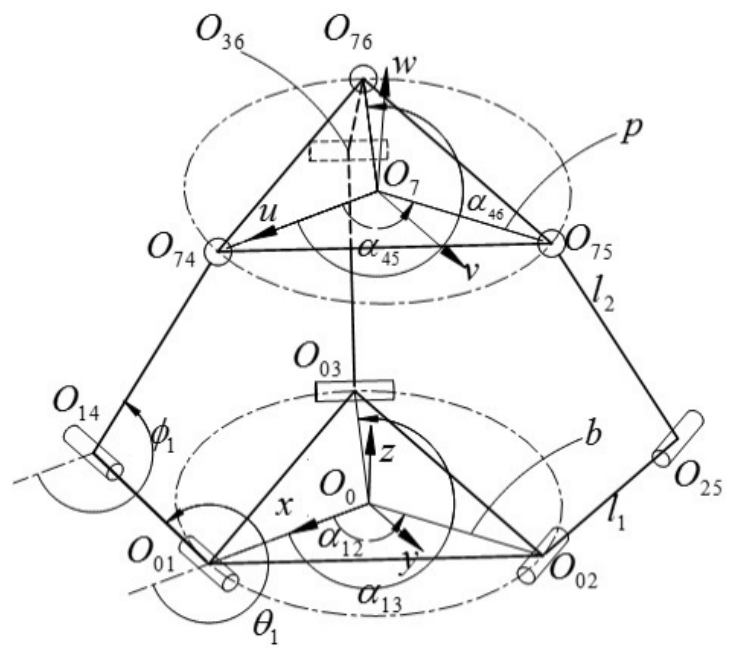

Fig. 1 Geometry of the 3-RRS parallel manipulator

To define the platform orientation, a rotation matrix is generated using $x-y-z$ Euler rotation sequence with orientation angles $\psi_{x}, \psi_{y}$ and $\psi_{z}$ of the platform:

$$
\mathbf{R}=\left[\begin{array}{ccc}
\mathrm{c} \psi_{y} \mathrm{c} \psi_{z} & -\mathrm{c} \psi_{y} \mathrm{~s} \psi_{z} & \mathrm{~s} \psi_{y} \\
\mathrm{~s} \psi_{x} \mathrm{~s} \psi_{y} \mathrm{c} \psi_{z}+\mathrm{c} \psi_{x} \mathrm{~s} \psi_{z} & \mathrm{c} \psi_{x} \mathrm{c} \psi_{z}-\mathrm{s} \psi_{x} \mathrm{~s} \psi_{y} \mathrm{~s} \psi_{z} & -\mathrm{s} \psi_{x} \mathrm{c} \psi_{y} \\
\mathrm{~s} \psi_{x} \mathrm{~s} \psi_{z}-\mathrm{c} \psi_{x} \mathrm{~s} \psi_{y} \mathrm{c} \psi_{z} & \mathrm{~s} \psi_{x} \mathrm{c} \psi_{z}+\mathrm{c} \psi_{x} \mathrm{~s} \psi_{y} \mathrm{~s} \psi_{z} & \mathrm{c} \psi_{x} \mathrm{c} \psi_{y}
\end{array}\right]
$$

where $\mathrm{c}$ and s stand for sine and cosine, respectively. Since the PM has 3-dof, three of the pose parameters given in Eqs. (1) and (2) are selected as independent task space parameters and the remaining three are found by solving the constraint equations. Considering the motion characteristics of the 3-RRS PM, the independent pose parameters are selected as $O_{7, z}, \psi_{x}$ and $\psi_{y}$. The constraint equations to obtain the dependent task space parameters are given in [8].

\section{Jacobian Analysis}

For the $i^{\text {th }}$ limb of the PM, the platform location can be expressed as:

$$
\overrightarrow{O_{0} O_{7}}+\overrightarrow{O_{7} O_{7 j}}=\overrightarrow{O_{0} O_{0 i}}+\overrightarrow{O_{0 i} O_{i j}}+\overrightarrow{O_{i j} O_{7 j}}
$$

By differentiating Eq. (3), the velocity loop equation for the $i^{\text {th }}$ limb is obtained: 


$$
\vec{v}_{O_{7}}+\vec{\omega}_{p} \times \overrightarrow{O_{7} O_{7 j}}=\left(\vec{y}_{i} \times \overrightarrow{O_{0 i} O_{i j}}\right) \dot{\theta}_{i}+\left(\vec{y}_{i} \times \overrightarrow{O_{i j} O_{7 j}}\right) \dot{\phi}_{i}
$$

where $\overrightarrow{y_{l}}=\left[\begin{array}{lll}-\mathrm{s} \alpha_{1 i} & \mathrm{c} \alpha_{1 i} & 0\end{array}\right]^{\mathrm{T}}, \overrightarrow{v_{O_{7}}}$ is the linear velocity of $O_{7}$ and $\overrightarrow{\omega_{p}}$ is angular velocity of the moving platform. The passive joint velocity on $i^{\text {th }} \operatorname{limb}, \dot{\phi}_{l}$, should be eliminated from Eq. (4) in order to obtain the relation between input and output velocities. Taking the scalar product of both sides of Eq. (4) with $\overrightarrow{\mathrm{O}_{l \jmath} \mathrm{O}_{7 \mathrm{~J}}}$ and rearranging:

$$
\overrightarrow{O_{i j} O_{7 j}} \cdot \vec{v}_{O_{7}}+\left(\overrightarrow{O_{7} O_{7 j}} \times \overrightarrow{O_{i j} O_{7 j}}\right) \cdot \vec{\omega}_{P}-\left(\overrightarrow{O_{0 i} O_{i j}} \times \overrightarrow{O_{i j} O_{7 j}}\right) \cdot \vec{y}_{i} \dot{\theta}_{i}=0
$$

Three of the velocity components, $v_{O_{7 x},}, v_{O_{7, y}}, \omega_{z}$, depend on the task space velocities $\omega_{x}, \omega_{y}$ (there is no dependency on $v_{O_{7, z}}$ ) as [7]:

$$
\left.\begin{array}{c}
v_{O T, x}=g_{x x} \omega_{x}+g_{x y} \omega_{y}=-\frac{p \mathrm{c} \psi_{y} \mathrm{~s} \psi_{y}\left(\mathrm{c} \psi_{x}+\mathrm{c} \psi_{y} \mathrm{~s} \psi_{x} \mathrm{~s} \psi_{y}\right)}{2\left(1+\mathrm{c} \psi_{x} \mathrm{c}_{y}\right)^{2}} \omega_{x}-\frac{p \mathrm{c} \psi_{y} \mathrm{~s} \psi_{x}\left(\mathrm{c} \psi_{x}+\mathrm{c} \psi_{y}+\mathrm{s} \psi_{x} \mathrm{~s} \psi_{y}\right)}{2\left(1+\mathrm{c} \psi_{x} \mathrm{c}\right)^{2}} \omega_{y} \\
v_{O T, y}=g_{y x} \omega_{x}+g_{y y} \omega_{y}=-\frac{p \mathrm{~s} \psi_{x} \mathrm{~s} \psi_{y}\left(\mathrm{c} \psi_{x}+\mathrm{c} \psi_{y}\right)}{\left.\left(1+\mathrm{c} \psi_{x} \mathrm{c}_{y}\right)^{2} \mathrm{~s}^{4} \psi_{y}-\mathrm{c}^{4} \psi_{y}+\mathrm{c}^{2} \psi_{x} \mathrm{c} 2 \psi_{y}-\right)}\left(\frac{\mathrm{c} \psi_{x}}{4}\left(5 \mathrm{c} \psi_{y}+3 \mathrm{c} 3 \psi_{y}\right)\right.
\end{array}\right) \omega_{y}
$$

Substituting Eq. (6) in Eq. (5) for all limbs results in:

$$
\mathbf{J}_{X}\left[\begin{array}{c}
v_{O 7, z} \\
\omega_{x} \\
\omega_{y}
\end{array}\right]+\mathbf{J}_{Q}\left[\begin{array}{c}
\dot{\theta}_{1} \\
\dot{\theta}_{2} \\
\dot{\theta}_{3}
\end{array}\right]=\left[\begin{array}{c}
0 \\
0 \\
0
\end{array}\right]
$$

where

$$
\mathbf{J}_{Q}=\left[\begin{array}{ccc}
\left(\overrightarrow{O_{01} O_{14}} \times \overrightarrow{O_{14} O_{74}}\right) \cdot \vec{y}_{1} & 0 & 0 \\
0 & \left(\overrightarrow{O_{02} O_{25}} \times \overrightarrow{O_{25} O_{75}}\right) \cdot \vec{y}_{2} & 0 \\
0 & 0 & \left(\overrightarrow{O_{03} O_{36}} \times \overrightarrow{O_{36} O_{76}}\right) \cdot \vec{y}_{3}
\end{array}\right]_{3 \times 3}
$$




$$
\mathbf{J}_{X}=\left[\begin{array}{lll}
A_{1}^{z} & A_{1}^{x} g_{x x}+A_{1}^{y} g_{y x}+B_{1}^{x}+B_{1}^{z} g_{z x} & A_{1}^{x} g_{x y}+A_{1}^{y} g_{y y}+B_{1}^{y}+B_{1}^{z} g_{z y} \\
A_{2}^{z} & A_{2}^{x} g_{x x}+A_{2}^{y} g_{y x}+B_{2}^{x}+B_{2}^{z} g_{z x} & A_{2}^{x} g_{x y}+A_{2}^{y} g_{y y}+B_{2}^{y}+B_{2}^{z} g_{z y} \\
A_{3}^{z} & A_{3}^{x} g_{x x}+A_{3}^{y} g_{y x}+B_{3}^{x}+B_{3}^{z} g_{z x} & A_{3}^{x} g_{x y}+A_{3}^{y} g_{y y}+B_{3}^{y}+B_{3}^{z} g_{z y}
\end{array}\right]
$$

with $\overrightarrow{O_{\imath \jmath} O_{7 \jmath}}=\left[A_{i}^{x} A_{i}^{y} A_{i}^{z}\right]^{T}$ and $\overrightarrow{O_{7} O_{7 \jmath}} \times \overrightarrow{O_{\imath \jmath} O_{7 \jmath}}=\left[B_{i}^{x} B_{i}^{y} B_{i}^{z}\right]^{T}$.

\section{Inverse Kinematics Singularities}

The inverse kinematics singularity condition is satisfied if $\operatorname{det}\left[\mathbf{J}_{Q}\right]=0$, or equivalently if $\overrightarrow{O_{0 \imath} O_{l \jmath}} \times \overrightarrow{O_{\imath \jmath} O_{7 \jmath}}=\overrightarrow{0}$ for any limb $i$. This configuration refers to the positions when the bottom $\left(\overrightarrow{O_{0 \imath} O_{l \jmath}}\right)$ and upper $\left(\overrightarrow{O_{l \jmath} O_{7 \jmath}}\right)$ links on limb $i$ are collinear. Such a configuration is obtained when a double root is obtained during the inverse kinematics solution where two different assembly modes of a limb converges to each other. Instead of working with the determinants of Jacobian matrices, the IKS can geometrically be expressed in terms of the task space parameters using the collinerarity condition of the limbs. Since all limbs of the PM have a planar motion, local planar coordinate frames $\left(O_{0}-x^{i} z\right)$ can be attached to each limb $i$ (Fig. 2).

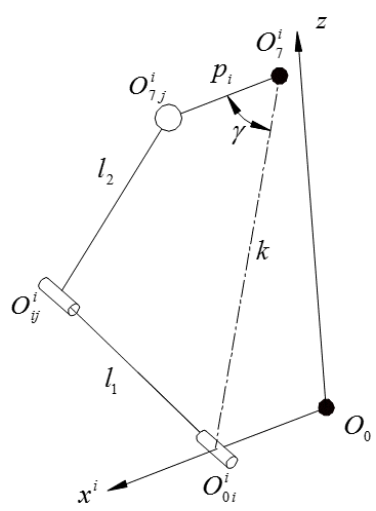

Fig. 2 Geometry of the 3-RRS parallel manipulator

The global coordinates of any point $\mathrm{P}$ on the $\mathrm{PM}$ can be projected onto the local planar coordinates as:

$$
\overrightarrow{O_{P}}=\left[\begin{array}{lll}
O_{P, x} & O_{P, y} & O_{P, z}
\end{array}\right]^{T} \rightarrow \overrightarrow{O_{P}^{i}}=\left[\begin{array}{ll}
O_{P, x} \mathrm{c} \alpha_{1 i}+O_{P, y} \mathrm{~s} \alpha_{1 i} & O_{P, z}
\end{array}\right]^{T}
$$

The lengths $p^{i}$ and $k$ (projections of $\overrightarrow{O_{7} O_{7 j}}$ and $\overrightarrow{O_{0 \imath} O_{7 j}}$ on the $x^{i} z$-plane), and the angle $\gamma$ in Fig. 2 can be expressed in tems of task space parameters as: 


$$
p^{i}=\left|\overrightarrow{O_{7}^{i} O_{7 j}^{i}}\right|, k=\left|\overrightarrow{O_{0 i}^{i} O_{7}^{i}}\right|, \cos \gamma=\left(\overrightarrow{O_{7}^{i} O_{7 j}^{i}} \cdot \overrightarrow{O_{7}^{i} O_{0 i}^{i}}\right) / p^{i} k
$$

The collinearity condition of the bottom and upper links of limb $i$ can geometrically be expressed by making use of the cosine theorem for $O_{0 i}^{i} O_{7 j}^{i} O^{i}{ }_{7}$ triangle:

$$
\cos \gamma=\left[p_{i}^{2}+k^{2}-\left(l_{1}+l_{2}\right)^{2}\right] /\left(2 p_{i} k\right)
$$

Eq. (12) is written for the extended dead-center configuration of the links. For the folded dead-center configuration, $l_{1}+l_{2}$ in Eq. (12) should be replaced by $l_{2}-$ $l_{1}$, but this case never occurs for our manipulator due to link collisions. Combining Eqs. (11) and (12), the inverse kinematics singularity equation for the 3-RRS PM can be formulated in terms of the task space parameters as:

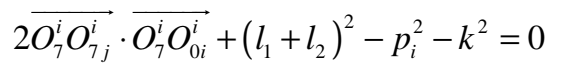

Eq. (13) represents a surface in the task space parametrized by $\psi_{x}, \psi_{y}$ and $O_{7, z}$. Using Mathematica $^{\circledR}$, the IKS surface given by Eq. (13) for all the limbs of the 3RRS PM can be constructed (Eq. (13) is written three times for $i=1,2,3)$. Considering the motion characteristics of the PM, it is preferred to plot the surface in spherical coordinates: $O_{7, z}=\sqrt{x^{2}+y^{2}+z^{2}}$ (in meters), $\psi_{x}=\operatorname{atan} 2\left(-\sqrt{x^{2}+y^{2}}, y\right)$ and $\psi_{z}=\operatorname{atan} 2(z, x)$. The IKS surface of the 3-RRS PM is presented in Fig. 3a. The singularity manifold has mirror symmetry about the xz-plane. In order to illustrate the contours inside, a cross section for $z=1$ is given in Fig. 3b.
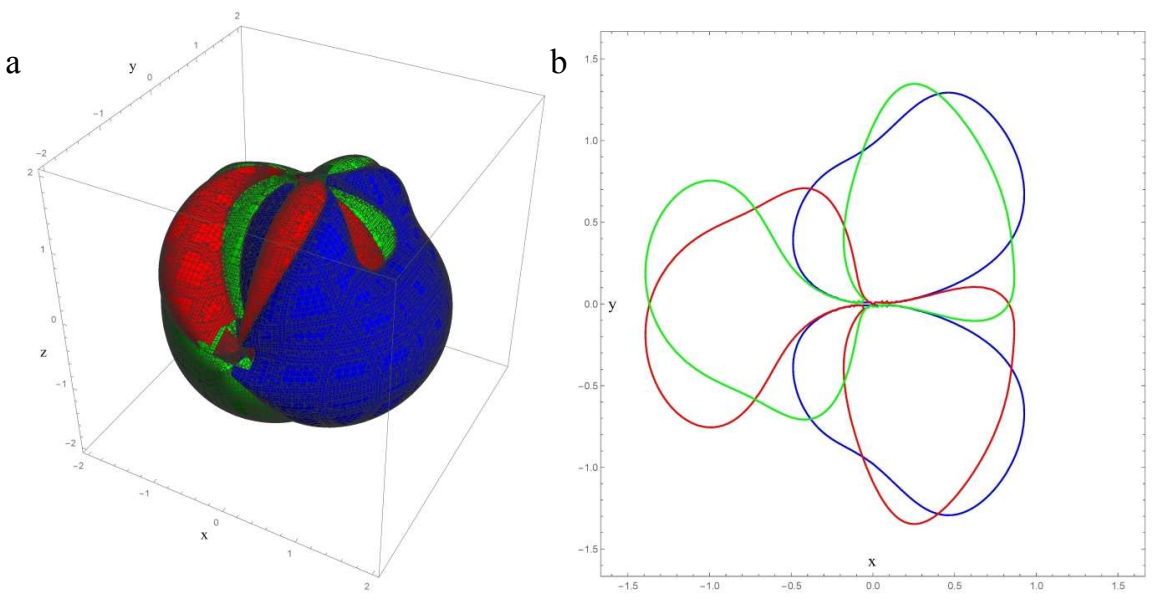

Fig. 3 IKS surface: a) Full view, b) $z=1$ section view 


\section{Forward Kinematics Singularities}

The forward kinematic singularities of the 3 -RRS PM are obtained $\operatorname{det}\left[\mathbf{J}_{X}\right]=0$. It is not straightforward to extract the geometrical conditions which make the determinant zero. However, when the $\operatorname{det}\left[\mathbf{J}_{X}\right]=0$ is formulated and plotted, it is seen that the singular configurations correspond to the cases where at least one of the upper links of a limb is coplanar with the platform plane, i.e. $\overrightarrow{O_{7} O_{7 j}} \times \overrightarrow{O_{l j} O_{7 j}}=\overrightarrow{0}$, or equivalently $B_{i}^{x}=B_{i}^{y}=B_{i}^{z}=0$ for any $i=1,2,3$. Coplanarity of an upper link and the platform corresponds to a double root during the forward kinematics solution. Instead of evaluating the Jacobian determinant, the coplanarity condition can be expresses in the local coordinate frame of the $i^{\text {th }}$ limb using cosine theorem for $O^{i}{ }_{0 i} O_{i j}^{i} O^{i}{ }_{7}$ triangle (Fig. 2):

$$
\cos \gamma=\left[\left(p_{i}+l_{2}\right)^{2}+k^{2}-l_{1}^{2}\right] /\left[2 k\left(p_{i}+l_{2}\right)\right]
$$

Singularity also occurs when the upper link is folded on the platform, in which case the $l_{2}+p_{i}$ term should be replaced by $l_{2}-p_{i}$. Combining Eqs. (11) and (14), the FKS equation for a limb can be formulated in terms of the task space parameters as:

$$
2\left(p_{i}+l_{2}\right)\left(\overrightarrow{O_{7}^{i} O_{7 j}^{i}} \cdot \overrightarrow{O_{7}^{i} O_{0 i}^{i}}\right)-p^{i}\left[\left(p_{i}+l_{2}\right)^{2}+k^{2}-l_{1}^{2}\right]=0
$$

Using Mathematica ${ }^{\circledR}$, the singularity surface for all limbs is constructed in sphrecial coordinates (Fig. 4). Although the FKS surface is plotted in the task space, it is possible to plot the surface in the joint space using inverse kinematics formulation.
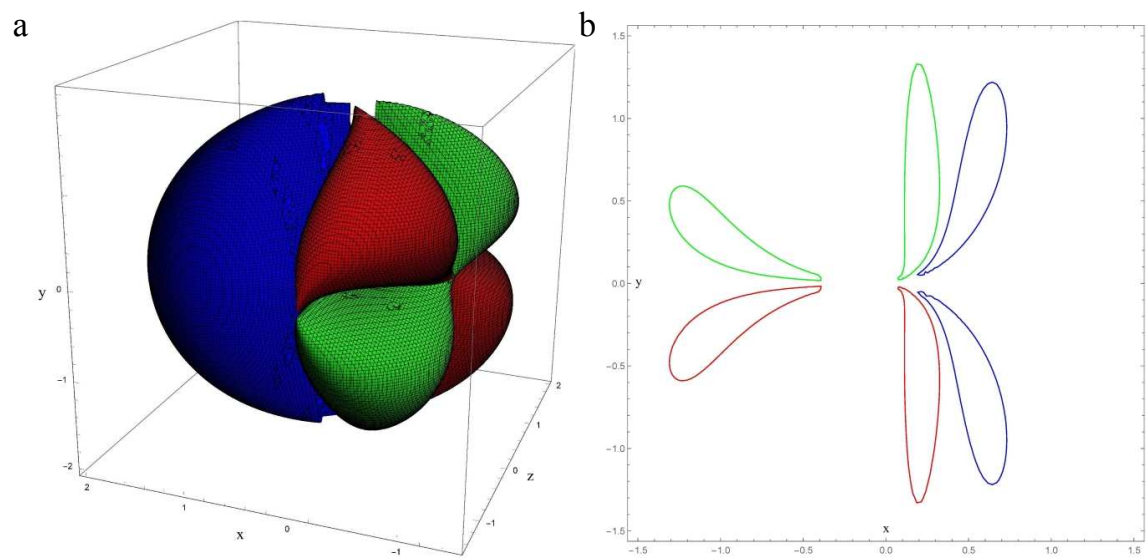

Fig. 4 FKS surface: a) Full view, b) $z=1$ section view 


\section{Conclusions}

This study presents a geometrical approach to determine the inverse and forward singularity manifolds of a 3-RRS PM. For the IKS, the Jacobian matrix $\mathbf{J}_{Q}$ is a diagonal matrix and each diagonal element being equal to zero corresponds to IKS configurations due to one of the limbs of the PM. However, it is not straightforward to express the diagonal elements in terms of the task space parameters. On the other hand, using the physical interpretation of the singularity condition and by simply writing a cosine theorem for each limb, the singularity surfaces can be very easily obtained in terms of the task space parameters.

In general, for the FKS of PMs, it is an hard task to relate the phisycal interpretation of the singular configurations with the determinant of the Jacobian matrix $\mathbf{J}_{X}$. For the 3-RRS manipulator issued in this study, although each of the three limbs independently contribute to the FKS, decomposing the Jacobian determinant into three is a challenging task. Instead of using the Jacobian matrix, the FKS surfaces due to each limb is constructed independently in this study by making use of the geometrical conditions which result in singularities.

Another contribution of this study is expressing the singularity surfaces of a 1T2R PM in spherical coordinates.

Acknowledgments The authors acknowledge the discussions with Prof. Sandipan Bandyopadhyay from IIT Madras, India.

\section{References}

1. Basu, D. and Ghosal, A.: Singularity analysis of platform-type multi-loop spatial mechanisms. Mech. Mach. Theory, 32, 375-389 (1997)

2. Chen, Q.H., Chen, Z., Chai, X.X. and Li, Q.C.: Kinematic analysis of a 3-axis parallel manipulator: the P3. Adv. Mech. Eng. (2013) http://dx.doi.org/10.1155/2013/589156

3. Gosselin, C. and Angeles, J.: Singularity analysis of closed-loop kinematic chains. Robot. Autom. IEEE Trans., 6, 281-290 (1990)

4. Li, Y. and Xu, Q.: Kinematic analysis of a 3-PRS parallel manipulator. Robot. Comput. Integr. Manuf., 23, 395-408 (2007)

5. Merlet, J.-P.: Parallel Robots. Springer (2001)

6. Rezaei, A., Akbarzadeh, A., Nia, P.M. and Akbarzadeh-T, M.R. Position, Jacobian and workspace analysis of a 3-PSP spatial parallel manipulator. Robot. Comput. Integr. Manuf, 29, 158$173(2013)$

7. Tetik H.: Modelling and Control of a 3-RRS Parallel Manipulator. MSc Thesis, İzmir Institute of Technology (2016)

8. Tetik, H., Kalla, R., Kiper, G. and Bandyopadhyay, S.: Position kinematics of a 3-RRRS parallel manipulator. In: Parenti-Castelli, V. and Schiehlen, W. (Eds.), ROMANSY 21 - Robot Design, Dynamics and Control, Springer, pp. 65-72 (2016)

9. Tsai, L.-W.: Robot Analysis: The Mechanics of Serial and Parallel Manipulators. John Wiley \& Sons (1999) 